\title{
Analytical Interference, Who Should Assess Kits?
}

\author{
Jude Joseph Fleming ${ }^{1}$
}

Published online: 13 May 2015

(C) Association of Clinical Biochemists of India 2015

In 2001 we published a paper in this journal outlining how individual laboratories could assess their own laboratory methods for endogenous interference from bilirubin, lipid and haemolysis [1].

Now 14 years later there is a much greater wealth of information about this besetting problem in Clinical Chemistry [2-4]. I will not consider interference problems in immunoassays [5] in this editorial, as it a major problem which deserves consideration in its own right. It appears that there is now also a debate about whose responsibility is it to assess manufactured kits for assay interference. Many large laboratories are now following ISO standard 15189:2012 "medical laboratories-requirements for quality and competence." This is the standard used by the National Accreditation Board for Testing and calibration laboratories (NABL) for accrediting laboratories in India. Under Section 5.5 examination processes, subsection 5.5.1.2 the standard says that "the laboratory should obtain information from the manufacturer confirming the performance characteristics of the procedure. The laboratory will independently verify through objective evidence that the performance claims for the examination procedure have been met."

Does this mean that every laboratory should be independently checking manufacturer's claims for assay interference when they purchase a kit? Definitely laboratory directors/supervisors are responsible for the quality of data that is reported from their laboratories. The standard wants to ensure that we implement an effective detection system

Jude Joseph Fleming

jdfleming@cmcvellore.ac.in

1 Department of Clinical Biochemistry, CMC Hospital, Vellore 632 004, Tamil Nadu, India to identify specimens with clinically important interferents and that we have in place a specific policy to prevent reporting of inaccurate results. A publication by Contois and Nguyen in 2012, reminds us that the laboratory is likely to see contradictory data when comparing in-house interference data to information reported on the package insert [6]. Since the quality of interference testing data provided by the manufacturer is seldom questioned, in-house interference testing is not normally performed. Visual inspection of samples alone may not be not effective in picking out haemolysed, icteric or lipaemic samples which can have an effect on the quality of results generated. The use of automated, spectrophotometric measurement of bilirubin, hemoglobin, and lipemia (serum indices), along with clearly defined decision rules, is available but how may laboratories make use of them? I think that this is because the serum indices programming takes out at least 3 channels on the instrument and inevitably reduces the number of tests performed per hour. One major figure, James Westgard says "we should do our own assessments. This is because many factors in your laboratory may be different from where the original assessment was performed. There may be the effects of shipment and storage, as well as local climate control conditions, quality of water, stability of electrical power, and of course, the skills of the analysts..." [7].

In my view it is unrealistic and unnecessary to expect small laboratories to perform inference studies on the kits they buy. They do not have the time or resources to do this and they have to rely on the manufacturer's supplied information. However the laboratory personnel should understand the mechanisms which produce interference in their assays and be prepared to resist demands by the requesting physician that they should "just give me the result". Top rank Clinical chemistry laboratories must also 
be prepared to be at the forefront in identifying new sources of analytical interference in close cooperation with the kit manufacturer.

So what should the small laboratory do if they find a suspiciously low or high analyte value in a patient which does not fit clinically? I will use the example of the patient has an HDL cholesterol and LDL cholesterol value both $<5$ with a total cholesterol value over $100 \mathrm{mg} / \mathrm{dl}$ and a normal/ high triglyceride value. We know that a set of results like this cannot be correct, so what should we do? Firstly repeat the analysis and confirm the values are consistent. Next rule out all possible pre-analytical errors. Make sure all relevant QC values are in range, that the kit has not expired etc., and that other specimens are giving correct results for those tests. Examine the specimen visually for the presence of clots, endogenous interferents, dilution effects etc. Confirm that it was collected in the appropriate container, and that it reached the laboratory in a timely manner. Ask are there any other abnormal values for the specimen which would indicate e.g. the presence of paraprotein, which can affect many assays [4]. Repeat the analysis on a different analyser, if available, preferably with a different manufacturer' kit. Small labs may have a neighbouring laboratory they can go to, and get the specimen re-analysed. Then, inform the requesting physician of the problem. Check with them that the patients was not on any infusion, and that the specimen was not taken inappropriately e.g. from the drip arm. Try and get a list of what medications the patient was receiving. Verify on the internet that none of these medicines are known to affect the test result in question. Report the affected test as falsely low/high value, with the comment "query assay interference? Cause unknown". Keep a record of these anomalous results. Next time the patient comes back check and see if the values are still abnormal. Contact the kit manufacturer and inform them of the problem. Give them the kit batch numbers and ask them if any other users have reported problems with that particular batch number. Ask them if they are able to give any suggestions or solutions for you. Having done all this, in my opinion it is then the manufacturer's responsibility to investigate problems with their reagents in the field. They may ask you to send them the specimen, so make sure you store it frozen, preferably at $-20{ }^{\circ} \mathrm{C}$ or even better at $-70{ }^{\circ} \mathrm{C}$. Send them as much clinical information as possible (this again may not be possible) listing other abnormal values glucose, electrolytes, creatinine, bilirubin etc. Do not assume that analysers costing 1 core rupees and more are any better at avoiding erroneous results from assay interference, since the problem is probably with the method principle employed and the kit reagent composition, not with the analyser.

There is a wealth of literature on the effect of drugs on tests, on interference in peroxidase assays (the Trinder method) and interference in creatinine estimation to name but a few. There are also CLSI guidelines on identifying interference in Clinical Chemistry assays [8]. Unfortunately the CLSI documents are not available on the internet without purchase. It is a great pity that valuable documents which could be used to raise our awareness of problems and direct us to solutions are not readily available in the public interest. The guidelines say that interfering substances may originate from the following endogenous and exogenous sources: metabolites produced in pathological conditions, compounds introduced during patient treatment; substances ingested by the patient; substances added during sample preparation; contaminants introduced during sample handling; the sample matrix itself.

We know we have a major problem with interferents in Clinical Chemistry kits and the laboratories should do their best, according to their resources, to work in partnership with the concerned manufacturer's to create awareness and devise solutions for these problems.

\section{References}

1. Fleming JJ, Swaminathan S. Interference in autoanalyser analysis. Indian J Clin Biochem. 2001;16:22-30.

2. Abstracts of the Scientific Posters, 2012 AACC Annual Meeting. Factors affecting test results. Clin Chem Supplement 2012;58: Poster A-10 to A-75, p. A14-A25.

3. Bowen RAR, Remaley AT. Interferences from blood collection components in clinical chemistry assays. Biochem Med. 2014;24:31-44.

4. Dalal BI, Brigden ML. Factious biochemical measurements resulting from haematologic conditions. Am J Clin Pathol. 2009;131:195-204.

5. Sturgeon MS, Viljoen A. Analytical error and interference in immunoassay: minimizing risk. Ann Clin Biochem. 2011;48: 418-32.

6. Contois JH, Nguyen R-A. Assay interference: a need for increased understanding and testing. LLC, New Gloucester: Sun Diagnostics, LLC: Sun Diagnostics; 2012. www.sundiagnostics.us and www. sundiagnostics.us/assuranc.

7. Westgard J. www.westgard.com/basic-method-validation-3rd-edi tion-faqs.htm.

8. CLSI. Interference testing in clinical chemistry; approved guidelines-second edition. CLSI document EPO7-A2. Wayne: Clinical and laboratory standards Institute; 2005. 